\title{
Developing Prognostic Models Using Duality Principles for DC-to-DC Converters
}

\author{
Mohammad Samie, Suresh Perinpanayagam, Member, IEEE, Alireza Alghassi, Student Member, IEEE, \\ Amir M. S. Motlagh, and Epaminondas Kapetanios
}

\begin{abstract}
Within the field of Integrated System Health Management, there is still a lack of technological approaches suitable for the creation of adequate prognostic model for large applications whereby a number of similar or even identical subsystems and components are used. Existing similarity among a number of different systems, which are comprised of similar components but with different topologies, can be employed to assign the prognostics of one system to other systems using an inference engine. In the process of developing prognostics, this approach will thereby save resources and time. This paper presents a radically novel approach for building prognostic models based on system similarity in cases where duality principle in electrical systems is utilized. In this regard, unified damage model is created based on standard Tee/Pi models, prognostics model based on transfer functions, and remaining useful life (RUL) estimator based on how energy relaxation time of system is changed due to degradation. An advantage is that the prognostic model can be generalized such that a new system could be developed on the basis and principles of the prognostic model of other systems. Simple electronic circuits, dc-to-dc converters, are to be used as an experiment to exemplify the potential success of the proposed technique validated with prognostics models from particle filter.
\end{abstract}

Index Terms-Cuk converter, degradation, duality, integrated system health management (ISHM), prognostic model.

\section{INTRODUCTION}

$\mathbf{T}$ HE next evolutionary step in condition based asset management is Integrated System Health Management (ISHM) [1] that attempts to enhance the reliability and safety of systems in critical environments by predicting, detecting, and mitigating undesirable events caused by degradation, fatigue and faults in heterogeneous distributed system (HDS) [2]. In this regard, effective estimation of the remaining useful life of devices and systems rely on the development of prognostic models. This thereby requires extensive efforts to be made toward creating suitable degradation profiles for every subsystem and its components. This is important because if only one component is upgraded, then a new degradation profile will be once again required. Consequently, changes committed to the design of the system will both consume time and incur additional cost, given that the prognostic models will need to be re-upgraded [3].

Manuscript received April 13, 2014; accepted November 11, 2014. Date of publication December 2, 2014; date of current version December 23, 2014. Recommended for publication by Associate Editor M. A. Huque.

M. Samie, A. Alghassi and S. Perinpanayagam are with the School of Applied Sciences, IVHM Centre, Cranfield University, Bedford MK43 0AL, U.K. (e-mail:m.samie@cranfield.ac.uk; a.alghassi@cranfield.ac.uk; suresh.nayagam @ cranfield.ac.uk).

A. M. S. Motlagh and E. Kapetanios are with the Faculty of Science and Technology, University of Westminster, London W1W 6UW, U.K. (e-mail: w141875@my.westminster.ac.uk; E.Kapetanios@westminster.ac.uk).

Digital Object Identifier 10.1109/TPEL.2014.2376413
To effectively overcome these problems, at the highest system level, a system-level reasoning (SLR) can be developed to at least provide the system with significant capabilities that can potentially decrease costs by assigning the system prognostics with a system-integrated prognostic reasoner (SIPR) [1], [4]. A vehicle-integrated prognostic reasoner (VIPR), for instance, is a NASA-funded effort for developing the next generation VLRS. A typical functional module within the SLR is a system reference model. This system reference model divides the system into partitions and provides the necessary relationships between subsystems for the inference process. This partitioning enables the inference engine to reuse and link the same prognostic models to multiple subsystems and further minimize certification and qualification costs [1], [4].

Although various techniques and methods, such as fuzzy [5], neural network [6], statistics, etc., have been utilized for the development of ISHM; it still suffers from problems related to inefficient models, uncertainties, and inadequate reasoning as well as being very costly and having time-consuming processes. These problems remain to be addressed, mainly because the prognostics of a system relies heavily on the physics of failure models and degradation profiles that are known to be either inaccurate, inconsistent, or very noisy. We believe that the ISHM system will greatly benefit if the prognostics of a component and a system are perceived as a feature of system rather than a component's physics of failure. An advantage of this approach is that it will enable SLR to develop prognostics for a new subsystem based on a collection of features (encompassing various models/patterns) already known from the previous prognostics of subsystems. In order to fulfill this task, SLR may need to employ similarity measurement and enhanced semantic search techniques in its inference engine and system reference model units. These techniques are used to measure similarities among a number of different subsystems and to automatically search which subsystem best matches the other related subsystems. Then SLR maps the subsystems properties to one another, in order to develop its prognostic model. For instance, Fig. 1 shows four different dc-to-dc converters, all with similar components but different topologies and functions. From a systemlevel point of view, these converters may present similarities in their system-level prognostics, since they all are built from similar components and because they develop similar degradation and failure mechanisms under different working conditions. Therefore, it can be assumed that SLR can link prognostics of these systems to other systems; that, in turn, saves the time and resources needed to develop prognostic models for every system. By using ac and dc circuit theory, the three basic converters 


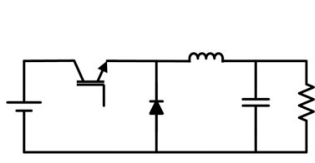

(a)

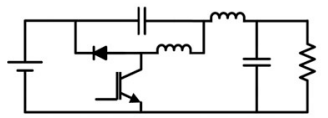

(b)

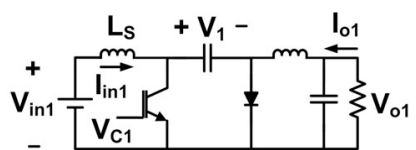

(c)

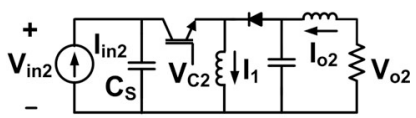

(d)
Fig. 1. Four different dc-to-dc converters: (a) voltage buck; b) buck-flipped; (c) Cuk; (d) dual of Cuk (D-Cuk) [8].

can be transformed to yield all known transfer functions and further realize a proliferation of 33 topologies. The circuit-based systematic unifying approach offers a novel insight and an alternative interpretation to the origin of 33 topologies including the Zeta, Cuk, \pm Luo, CSC, Landsman, and Sepic converters [7]. This means, that only a few unified prognostic models can be used by SLR to cover the prognostics of all 33 different topologies of converters. A similar unifying approach in interoperation systems can be identified in other electrical and electronic systems such as electrical motors, filters, etc. So by having similarities in functions, topologies, components and features will enlighten us on how the prognostics of a system can be fused from the prognostics of other systems.

In this paper, we employ a principle of duality in physics (and circuit and system theories) to assign prognostics of a circuit to its dual version. How we intend to do this is by developing a prognostic model using mathematical modeling of physical systems, graph, and duality theories of a subsystem's topology rather than individual parts of the components. In this regard, standard models provided by circuit and system theories are employed to develop unified damage model using data-driven approach; then transfer function from the unified model is used to develop prognostic model that predict the next failure state of system; finally remaining useful life (RUL) is estimated based on how energy of system is changed due to degradation over the time. For validation purposes, the results are compared with results from prognostic model created using model driven approach enhanced with particle filter. As a limitation, we consider that both a circuit under test and its dual version are comprised from the same components but connected in different topologies with different values as shown in Fig. 1(c) and (d) (in regards to Cuk converter and its circuit).

The duality concept has already been recommended for diagnosing faults. Reference [8] proposes a fault diagnoser based on the duality principle and the optimal control theory for linear systems. However, this paper will present duality applications in system prognostics. Our approach, which ultimately leads to exploiting novelty in system-level reasoning (SLR), is limited to development of a duality-based prognostic model applied to a single phase dc-to-dc Cuk converter and its dual version (D-Cuk) as a case study that presents the feasibility of similarity in linking prognostics of systems to one another.

In Section II, we shall further explain the prognostics of a system. Section III shall cover the principles of duality in electrical systems, along with brainstorming the duality concept of a system's prognostics. The prognostics of dc-to-dc converters with details of Cuk and D-Cuk circuits are explained in Section IV. An algorithm proposed that will help develop the prognostics for a dc-to-dc converter using a duality concept is illustrated in Section V. Section VI discusses the simulation results obtained via validation from Section VII. Lastly, Section VIII covers the conclusion.

\section{Prognostics}

In condition-based maintenance, prognostics can be defined as a controlled engineering discipline that focuses on predicting and estimating the future lifecycle of a system or component, i.e., establishing at what point the system/component starts to slowly develop irregularities and faults to the extent where it malfunctions. The predicted lifecycle of a system or component is referred to as the RUL, which is used for making decisions in regards to contingency mitigation and maintenance. There are various scientific techniques used that can help construct the prognostics of a system or a component, including failure mode analyses, early detection of aging signs, and damage propagation models. Failure mechanisms are often used in conjunction with system lifecycle management to create Prognostics and Health Management (PHM) disciplines.

\section{A. Technical Approaches}

There are four main technical approaches viable for building prognostic models: 1) data-driven approach that requires lengthy and costly accelerated aging tests and suffers from noisy data [10]-[12]; 2) model-based approach suffers from complexity and inaccuracies of physical and mathematical models [9], [13]; 3) hybrid approaches intend to bring the strength of the first two approaches into one prognostics strategy; however, this is very difficult to achieve for complicated systems [14]-[16]; and 4) service-based approaches refer to data that are collected during system maintenance. The accuracy related to these data remains to be questionable as it heavily depends on human interaction (i.e., human mistakes).

\section{B. Developing Prognostic Models}

Fig. 2 summarizes the basic knowledge needed to develop data-driven-based and model-based prognostics. The first approach uses a novel technique for creating system-level prognostics, while the other approach is used for validation purposes in this paper. As shown in Fig. 2, development of prognostic models is generally comprised from number of steps based on recognized techniques in data-mining approaches and RUL estimation methods. The process starts with a model definition step for model-based prognostics that require understanding the physics-of-failure of the component/system. As mentioned in the previous section, it is often a difficult task to establish an accurate physical model. On the other hand, data-driven approaches start with accelerating aging tests followed with preprocessing (filtering) and classification for eliminating noise from collected data and recognizing life durations of compo- 


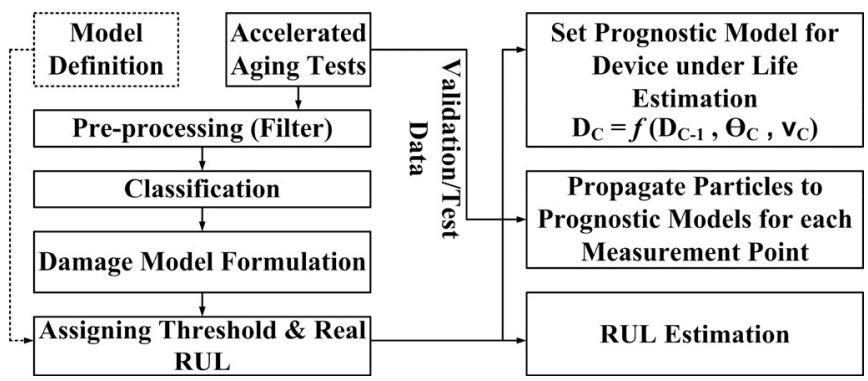

Fig. 2. Algorithm to develop prognostics model.

nent/system to health states wherever meaningful changes get observed from the collected data. There are various techniques to accelerate aging processes under different working/operational conditions, such as thermal cycling, power cycling, and electrothermal cycling [17], [18]. It is worth mentioning that there is also an important initial step that involves investigating what signals should be monitored to allow prognostics to be successfully developed based on their features extracted from those signals. This is known as feature extraction step.

Degradation of the component/system is then formulated (as a damage model) using various stochastic, probabilistic, soft computing methods. For instance, using a particle filter (PF) that is also known as the sequential Monte Carlo method [19], the previous damage state is used as the prior information to develop the current damage state, and the model parameters are updated by multiplying it with the likelihood. The damage model $(f)$ is given by the following equation:

$$
D_{C}=f\left(D_{C-1}, \theta_{C}, \nu_{C}\right)
$$

where $C$ is the cycle (or time step index) that refers to the specific cycle or time that the measurement data from the component/system has been taken, $D_{C}$ is the damage state at cycle $C, \theta_{C}$ is a vector of model parameters, and $\nu_{C}$ refers to process noise.

The created damage model is then used for obtaining the initial threshold, real/nominal RUL, and prognostic model. Suppose the component/system has been monitored by $n$ number of measurement points through its life period collected inside the validation/test data. Each measurement occurs at a specific time index or cycle $\left(C_{S}\right)$. RUL estimation is a process to sweep $C_{S}$ from measurement points 1 to $n$, and for each particular $C_{S}$ ( $S$ between 1 and $n$ ), estimate when degradation in the system is progressed to a threshold level $(T)$ where the system is aged enough to be known as a damaged system. Then RUL for each $C_{\mathrm{S}}$ is accounted as numbers of iterations that need to be performed from the initial $C_{S}$ to the point that the damaged state propagates to the threshold $D_{T}$. Based on how degradation is processed in the model, threshold may occur at different cycles $\left(C_{T}\right)$ for different measurement points $\left(C_{S}\right)$ so that $C_{T} \geq C_{S}$. For each measurement point (cycle), the distribution of model parameters accounted from previous cycles (measurement points) is expressed as a number of particles and their weights [20], [21]; and then, they are propagated toward
TABLE I

DUALITY PRINCIPLE IN ELECTRICAL SYSTEMS

\begin{tabular}{lc}
\hline \hline System & Dual of System \\
\hline Voltage of nodes or across device & Current of branch or mesh \\
Current of branch or mesh & Voltage of nodes or across device \\
Resistor $(R)$ & Conductivity $(1 / R)$ \\
Capacitor $(C)$ & Inductance $(L=C)$ \\
Voltage source $\left(V_{s}\right)$ & Current source $\left(I_{s}=V_{s}\right)$ \\
Kirchhoff's current law & Kirchhoff's voltage law \\
Mesh/loop & Node \\
\hline \hline
\end{tabular}

the threshold level. This process is continued from the first measurement point $\left(C_{S}=C_{1}\right)$ to where $C_{S}=C_{T}$.

\section{Prognostics of Dual Systems}

One of the fundamental properties of systems is duality. This can be consistently observed in systems that have any kind of physics [22], [23]. This has many implications within mathematics, engineering, and science. Duality relations have been identified between geometric objects, algebraic structures, topological constructs, and various other scientific constructs. In regard to electrical systems, duality relations have appeared in the core principles for any theorem within an electrical circuit analysis, for situations where there is a dual theorem that replaces one of the quantities with dual quantities. Examples of dual quantities in electrical systems are current and voltage, impedance and admittance, meshes, nodes, etc., found in electrical systems (shown in Table I) [24].

In regard to duality concepts, if the values of the parameters and topologies of both circuits are linked to one another based on the details shown in Table I, a duality relationship is expected to be found between two electrical circuits. So from a mathematical perspective, dual circuits are known to have the same mathematical model, apart from their parameters differing. Thus, while the functions of the systems are different, their prognostics can still be assigned to each other on the basis that dual relationships are found among the systems, along with the systems having the same mathematical model with dual parameters shown in Table I. This provides us with the required facilities needed to develop the prognostics of a system based on the prognostics of its dual system.

The functionality of a system is well established via graph theory [22], which illustrates that if the topology of a system is known, the components and devices used in the system need not to be known, given that the nodes voltages and currents of the branches in the circuits are already known. We can, therefore, expect that graph theory will provide us with the information required to construct the prognostics of a system based on its topology rather than concentrating on the integrated devices and components within the system. We can expect that systems sharing similar or dual topologies and mathematical models are likely to share similar prognostics, regardless of the components integrated within the system, thus making it possible to investigate how prognostic models can be constructed from knowing the topology of system rather than having to know the physics 


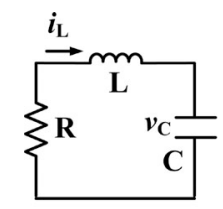

(a)

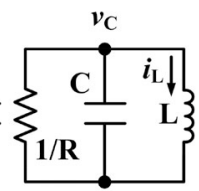

(b)

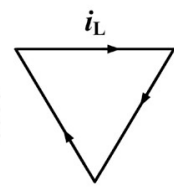

(c)

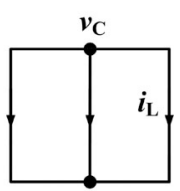

(d)
Fig. 3. (a and b) $R L C$ circuit with duality relationship; (c and d) graphs for circuits (a) and (b).

of failure of a system. As a result, this makes the process of modeling the prognostics of a system much more feasible and realistic, considering that it saves a substantial amount of time and resources. This is because it avoids shaving to individually test every system to identify its prognostics.

Fig. 3 shows an example of dual $R L C$ circuits. Using Kirchhoff's laws in the form of state equation, it can be said that both circuits have the same mathematical model as shown in (1) for circuit in Fig. 3(a) and (2) for circuit in Fig. 3(b)

$$
\begin{aligned}
& {\left[\begin{array}{l}
d i_{L} / d t \\
d v_{C} / d t
\end{array}\right]=\left[\begin{array}{cc}
-R / L & -1 / L \\
1 / C & 0
\end{array}\right]\left[\begin{array}{l}
i_{L} \\
v_{C}
\end{array}\right]} \\
& {\left[\begin{array}{l}
d v_{C} / d t \\
d i_{L} / d t
\end{array}\right]=\left[\begin{array}{cc}
1 / L & 0 \\
-1 / R C & -1 / C
\end{array}\right]\left[\begin{array}{l}
v_{C} \\
i_{L}
\end{array}\right] .}
\end{aligned}
$$

For instance, assuming that a degradation mechanisms is added, $R$ within the circuit of Fig. 3(a) is aged toward a short circuit $(R \rightarrow 0)$, this is turned to a degradation toward an open circuit $(1 / R \rightarrow \infty)$ in the circuit of Fig. 3(b). This represents the duality principles shown in Table I, thus proving that the resistor $(R)$ is a dual of a conductive $(1 / R)$; or in regard to this example, it can be known as the short circuit being the dual of an open circuit.

Similar rules can be applied to more complicated circuits as well, which involve using various components, including capacitors and inductances. The most critical point that must be considered refers to the degradation and failure mechanisms of dual components that are not completely related to one another. For instance, the degradation mechanism of a capacitor is not, in any shape or form, related to degradation mechanisms of inductance.

A solution to this problem would be to rely on Ohm's and Kirchhoff's laws of physics principles. These two well-known laws establish that any electric component can be formulated by using the voltage across the component and the current through the component. An alternative solution would be to use the basic principles related to graph theory's circuit and system design, considering that the behavior of a system is fully formulated if the voltage of all nodes and the current through all the branches in the circuit are also known. This means that the behavior and the function of a circuit can be fully formulated regardless of what components are used in the circuit, as long as all the voltages and currents are known. Fig. 3(c) and (d) shows the graph of the equivalent circuits in Fig. 3(a) and (b).
From a circuit-level point of view, the details required for the developing the prognostic model for a circuit does not necessary need to be known. We essentially use sensors for measuring voltages, currents, temperature, etc. So if we were to base it on the meaning of the sensed values, it would allow us to experience the level of degradation related to a circuit or system of any form, to be interpreted as a circuit not functioning properly. Although this principle can be applied for greater purposes, for example, to design a device-independent prognostic model, this paper will for now mainly concentrate on presenting a realization of duality principles for the development of prognostics for dual circuits.

\section{DC-TO-DC CONVERTER AND ITS DOMINANT FAILURES}

A simple building block for power convertor type systems is the dc-to-dc converter. There are various dc-to-dc voltage and current converters that have different topologies. The following topologies can be defined algebraically [25]-[28], graphically [29], [30], or in a matrix form [31]-[33]. It is of significant interest to unify the converter's topological characteristics, relationships, and analysis [34]. In regard to health management, the aim would be to develop a basic structure, model, or concept that encapsulates all the converters, including their prognostics that may have derived from and been mapped from duality principles. This unified model can offer many advantages in developing condition-based monitoring and SLR.

On the basis of what has been already presented in [35], the underlying concepts related to basic converters can be unified with regard to duality principles and in relation to current- and voltage-source converters. Williams [35] used circuit transformations to unify the basic converters, ultimately showing that other converters are derivable transformation topologies of the basic converter.

This section shows the dominant failures of the Cuk converter, along with how they can be linked to the failures of the D-Cuk. The schematic of the Cuk converter and its dual circuit are presented in Fig. 1(c) and (d). So Cuk is a step-down/step-up converter that shares a similar switching topology with buck-boost converter, thereby presenting the voltage ratio of a buck-boost converter as [36]

$$
\frac{v_{o}}{v_{s}}=\frac{D_{s}}{1-D_{s}}
$$

where $v_{o}$ is the output voltage, $v_{g}$ is the input voltage, $D_{\mathrm{S}}$ is the duty cycle of the switch $t_{\mathrm{on}} /\left(t_{\mathrm{on}}+t_{\mathrm{off}}\right)$; and $t_{\mathrm{on}}$ and $t_{\mathrm{off}}$ are the durations for when the switch is on and off. Equation (3) is calculated based on the principles related to conservative energy. As shown by the equation, the output voltage can be controlled by maintaining the duty cycle of the switch. Depending on what type of switching scheme is used, output voltage can be either higher or lower than the input voltage. The state equations for Cuk converter are

$$
\begin{aligned}
x^{\prime} & =A x+B v_{g}+B_{c} d \\
v_{o} & =C_{x} \\
x & =\left[v_{2} v_{1} i_{2} i_{1}\right]^{\prime} .
\end{aligned}
$$




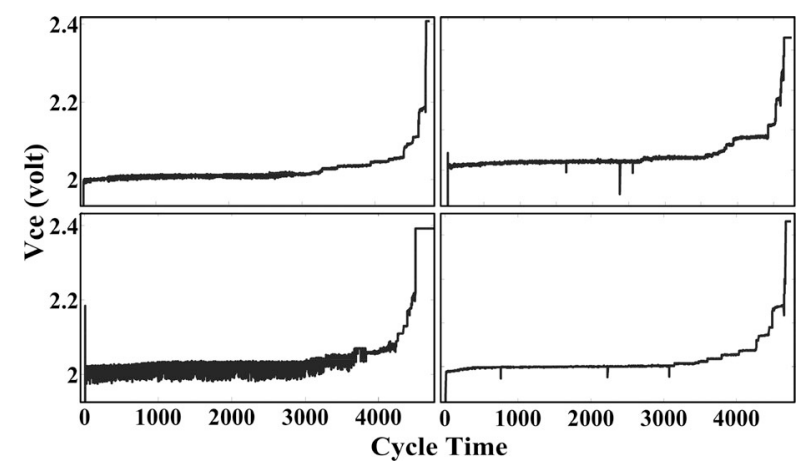

Fig. 4. Run to failure data for four different IGBTs.

The Cuk converter has two inputs, a control input $\left(V_{c}\right)$ and an input from the power supply $\left(v_{s}\right)$ along with one output $\left(v_{o}\right)$. In that sense, matrix $[A B C D]$ relates to "state-space matrices" for the open-loop model from the $v_{s}$ to the $v_{o}$. Similarly, $\left[A B_{c} C D\right]$ is the state space matrices from the control input $d$ to the output $v_{\mathrm{o}}$. Values for $A, B, B_{c}, C$, and $D$ are presented in [25]. The same equation can be extracted for the dual circuit of the Cuk converter in Fig. 1(d). However, their parameters are used in a dual form (shown in table I). $Y_{\text {in }}$ and $Z_{\text {in }}$ are the input admittance and input impedance of the Cuk circuit and its dual circuit.

The components found inside the converters that are mainly damaged refer to the IGBTs and capacitors. The results for the development of model-based prognostics related to a dc-to-dc power converter that focuses on the output filter capacitor component has been presented in [37] and [38]; however, this paper looks at the failure of converters caused by IGBTs. IGBTs experience a number of failure mechanisms, including bond wire fatigue, bond wire lift up, corrosion of the wires, static and dynamic latch up, loose gate control voltage, etc. We assume that such failure mechanisms can cause IGBTs to function either as an open circuit on a collector-emitter or a device encountering malfunction on its gate-emitter control. For instance, an IGBT's thermal junction is increased due to solder cracks, which become wire bond lift off, resulting to an increase in the resistor, relating to the collector-emitter. As for hot carrier injection, electrical stress is contrary increased, causing a short circuit on the IGBTs gate-emitter junction. This failure leads to the IGBT's gate controllability being missed (loose gate control voltage), causing the IGBT to not function. The result of this effect is that the current through collector-emitter is increased, meaning the resistor of the collector emitter is decreased. We can therefore establish that wire bond lift off and loose gate control voltage are failure mechanisms that have some kind of duality relationship. So while one of them increases the resistor, the other one decreases the resistor. This is why investigations must be conducted to identify how IGBT's failure mechanisms present a duality relationship to one another, which does not reflect with the aim of this paper. We thereby assume that IGBT's failure and malfunction mechanisms are parameters that have duality relationships.

Fig. 4 shows IGBT run to failure data relating to four different IGBTs. The following data are very noisy and need to be filtered.

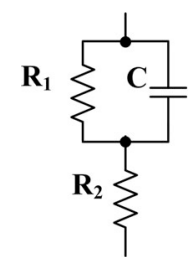

(a)

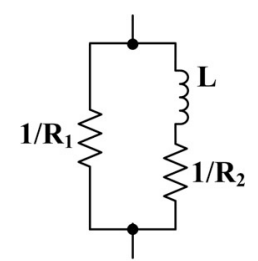

(b)
Fig. 5. Model for (a) capacitor and (b) inductance.

However a number of states still continue to exist that can be viewed. These states refer to cracks or wires that were lifted off due to degradation mechanisms. The resulting effects are changes in the IGBT's functionality and changes in the channel resistor of that IGBT. It is assumed that degradation is processed in a form of duality for the Cuk and its dual circuit, which means that if the IGBT of the Cuk experiences degradation toward its open circuit, IGBT of dual circuit of Cuk is degraded toward short circuit. By the time that the IGBTs are damaged, $C_{S}$ and $L_{S}$ are fully charged, as the other energy storage components lost energy, leads to $V_{o}$ hence becoming 0 . We assume that IGBT short circuit may have occurred when the current through the collector-emitter exceeded over its limit just before the IGBT was burned out.

Based on the level of accuracy, there are number of models that can be applied to a real capacitor and an inductance. In order to simplify a simulation, we assume that the capacitor and the inductance can both be modeled as in Fig. 5 for the purposes of this paper. The models describe above will present us with a duality relationship found between capacitance and inductance while also presenting the amount of energy lost by the resistors. $R_{1}$ typically has very large values, while $R_{2}$ has a small value; but due to degradation, these resistors are changed toward either open or short circuits.

\section{Algorithm to Develop Prognostics AND ESTIMATE RUL}

The proposed algorithm illustrated in Fig. 6 is devised to develop the damage model, prognostics model, and RUL estimator based on an algorithm shown in Fig. 2. Using this algorithm, the damage model is created based on a standard Tee/Pi model (see Fig. 7), the prognostic model is created based on its transfer functions; and finally, the RUL is estimated based on how the energy is lost or relaxed in the circuit.

\section{A. Damage Model Development}

The same process that possesses different sets of run to failure degradation and malfunction profiles is repeated for both the Cuk and its dual circuit. Initially, the components of the circuits are first set to be operating in good condition, then immediately after the time step for the circuit is increased, the components' values are changed via a series of values provided within the degradation profile for the new time step. At each time step phase, signals such as $v_{1}, v_{2}, v_{o}, i_{1}, i_{2}$, and $i_{o}$ are measured. The following signals are used for calculating the properties of the 


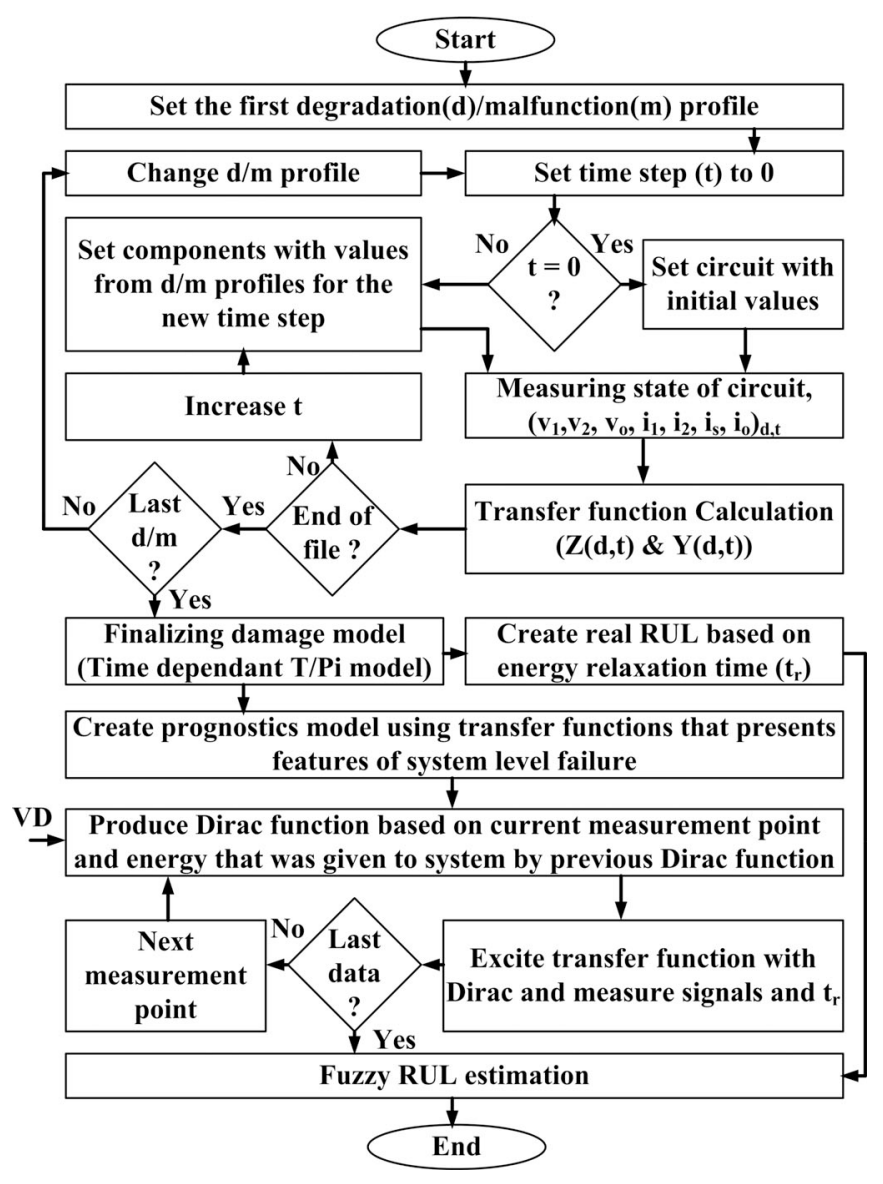

Fig. 6. Algorithm used to develop prognostic model: $d / m$, degradation/malfunction number; $t$, time step or cycle.

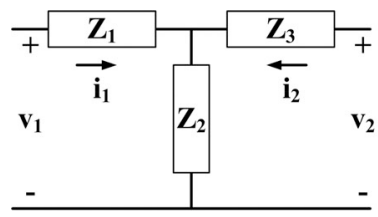

(a)

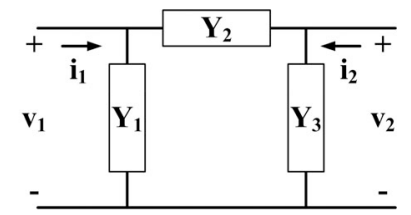

(b)
Fig. 7. Tee and Pi models.

system such as transfer functions, input and output impedances, and admittances. As a result of the changes encountered by the system during simulation, the system degradation is turned to the transfer functions $\left[Z_{c}(d, t), Y_{c}(d, t), Z_{\mathrm{dc}}(d, t), Y_{\mathrm{dc}}(d, t)\right]$. So where $d$ is an index of a selected degradation profile, $c$ and dc refer to Cuk and D-Cuk converters, respectively. Whenever $d$ is altered, the time step $(t)$ is reset to zero, which resets the process of the circuit for the new degradation scheme to a healthy condition.

The implication of such a process is to obtain standard Tee and Pi models for the Cuk and D-Cuk converter (as shown in Fig. 7). This implies that for each degradation and malfunction profile, there would be a number of time-dependent Tee and Pi models that are fused into a damage model. Various techniques, such as neural network, fuzzy, statistics, etc., among others, can be utilized, where out of all the time-dependent Tee/Pi models, one can generate a universal damage model for the converters (Cuk and D-Cuk). In this case, we use a mean value to simplify and speed up the process of fusing damage models. The created damage model is then analyzed to find which transfer function best presents the features of the circuit's failure. The chosen transfer function is used as a prognostic model by the RUL estimator to predict the future state of failure while it is being excited by validation and test data.

\section{B. Envisioning Features of System-Level Failure}

To envision the relevant features of failure determined from the damage model, the converter's time constant $\tau$ (referring to how fast the system is charged and discharged) is also measured from changes in $i_{1}$ for the Cuk converter $\left(\Delta i_{1}\right)$, and $v_{2}$ for D-Cuk $\left(\Delta v_{2}\right)$. Based on the principles of physics, $\tau$ is expressed as $R C$ and $L / R$ for capacitive and inductive circuits, respectively. In many circuits, for instance, the Cuk converter, we encounter many more complicated circuits that are modeled as $R L C$ circuit rather than just $R C$ or $R L$ circuits. However, $\tau$ does not apply to $R L C$ circuits as it does to $R C$ or $R L$ circuits; but instead, $\tau$ is capable of measuring how fast signals are damped in the circuit. As for a series-RLC circuit, for example, attenuation $\alpha$ is $R /(2 L)$ and damping factor $\zeta$ is $\alpha /\left(2 \pi f_{0}\right), \alpha$ is called the nipper frequency, or attenuation, which measures how fast the transient response of the circuit will die away once the stimulus is removed [39], [40]; $f_{0}$ is the angular resonance frequency that is $f_{0}^{2}=1 /\left(4 \pi^{2} L C\right)$. By using a duality concept, the attenuation factor can be calculated from $1 /(2 R C)$, for parallel- $R L C$ circuit.

The reason for measuring the aforementioned signals and parameters is that it would lead to establishing how energy is transferred between capacitances and inductances and how that transferred energy is lost when the system is also degraded. Then, the time taken for energy to be loss or relaxed is employed to estimate the RUL of the circuit. A circuit can be ultimately simplified as an $R L C$ circuit where the energy is frequently transferred between the capacitor and the inductor and causes the circuit to oscillate/resonate. In this case, the $Q$ factor is a widespread measure used to characterize resonators. It is defined as the peak energy stored in the circuit which is divided by the average energy dissipated in it per cycle at resonance. Thus, low $Q$ circuits are therefore damped and lossy and high $Q$ circuits are under damped. $Q$ is related to bandwidth $Q=\left(2 \pi f_{0}\right) / \mathrm{BW}$, with BW referring to bandwidth [22]. We expect that due to system degradation, the system-level parameters such as $\tau, f_{0}$, $\alpha$, and $\zeta$ and unwanted transient modes will be changed.

\section{RUL Estimation at System Level}

The RUL of both converters can be successfully estimated by repeating the same process for each measurement point of the test/validation data. In this regard, a real/nominal RUL is assigned to the damage model so that if the damage model (assumed as a black box) is excited by a Dirac function $\left(\alpha \delta\left(t-t_{s}\right)\right)$ at $t_{S}$ while having initial energy $E_{S-1}^{+}$, the response of the damage model would be a transfer function (prognostic model) at time index $t_{S}$, while the energy of the system is also 


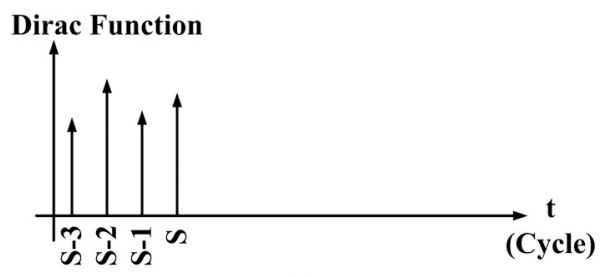

(a)

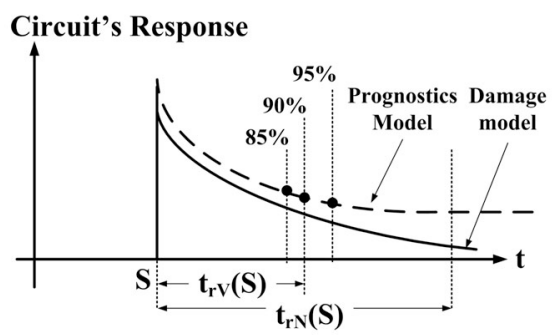

(b)

Fig. 8. Calculating RUL from response of circuit.

changed from $E_{S-1}^{+}$to $E_{S}^{+}$. $E_{S-1}^{+}$and $E_{S}^{+}$denote the energy of a system (stored in capacitors and inductors) just after $t_{S-1}$ and $t_{S}$, respectively. The Dirac function provides an impulse input of $\alpha$ for the converter over the period of $t_{S}>0$ so that circuit is energized at time $t_{S}$; and then, energy is relaxed or reduced over time. The time needed where $95 \%$ of saved energy has been lost is related to the prognostic model's parameters. If relaxation time is $t_{r}$, then the nominal/real RUL (known as $\mathrm{RUL}_{\mathrm{N}}$ ) for the measurement point at $t_{S}$ is related to $\operatorname{RUL}_{N}(S) \propto t_{\mathrm{r}}=t_{\mathrm{Th}}-t_{\mathrm{S}}$; and $t_{\mathrm{Th}}$ is accounted for when $90 \%$ of energy is lost from $E_{s}^{+}$ (see Fig. 8). Confidence level for RUL can be estimated based on $85 \%$ and $95 \%$ energy loss (see Fig. 8).

Since damage and prognostic models are time-dependent Tee/Pi model and transfer functions, the RUL accounted for current measurement point $(S)$ is different from the one accounted for previous measurement point $(S-1)$. Once the parameters for the damage model are adjusted for the nominal RUL, the prognostic model can be updated with test data. Then, the RUL for any specific test/validation data at time step $t_{s}\left(\mathrm{RUL}_{V}(S)\right)$ is estimated based on the time difference to relax (loss) energy in the damage model and the updated prognostic model $\left(\operatorname{RUL}_{V}(S) \propto\left(\Delta t_{\mathrm{r}}=t_{\mathrm{rV}}(S)-t_{\mathrm{rN}}(S)\right)\right)$.

To include the dynamics of systems, $\operatorname{RUL}_{V}(S)$ is adjusted with working conditions using a fuzzy RUL estimator. The transfer function is excited with the Dirac function and fuzzy values are then assigned to the time difference between energy relaxation time of damage and prognostics models. An advantage of estimating RUL based on the energy relaxation time difference $\left(\Delta t_{r}\right)$ is that the propagation step (see Fig. 2) is eliminated in the RUL estimation by a fuzzy input that feeds the $\Delta t_{r}$ to fuzzy base knowledge.

Thus, for instance, the fuzzy values represent whether there are small, medium, normal, transient, or big changes experienced at the output of the converters time constant, resonance frequency, attenuation, damping facture of measured signals $(\tau$, $\left.f_{0}, \alpha, \zeta\right)$, etc. The meaning of the term "normal" in the fuzzy set

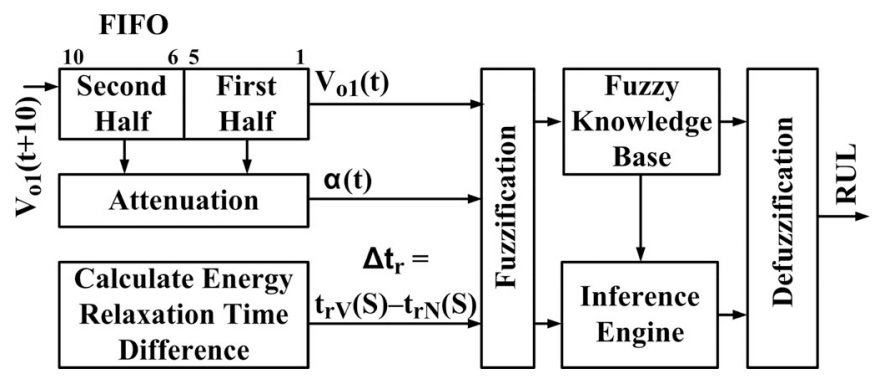

Fig. 9. Fuzzy-based RUL Estimator

refers to changes in the signals that can be ignored and transient means that the circuit is in a transient mode and should be settled in a steady state during a specific time constant.

In fuzzy-based RUL estimation, rules are set by experiments, whereby knowledge can be extracted from the changes experienced in the $\alpha$ and $Q$ that constitute for changes made to all those parameters previously mentioned, for example, bandwidth. $\alpha$ can be used to measure the time that it takes for the circuit to settle down to the steady-state value from when the transient mode is started to be accounted, directly from the measured signal. Then $Q$ is calculated using $\left(2 \pi f_{0} L\right) / R$ or $1 /\left(2 \pi f_{0} \mathrm{RC}\right)$. The advantage of having $Q$ involved in fuzzy rules is that the effects of degradation on $f_{0}$ and $\mathrm{BW}$ will be also accounted. Values of $R, L$, and $C$ in these equations are not the values that are directly available from the circuit shown in Fig. 1 or Pi- or Tee-based prognostic models (see Fig. 7) [22]. In order to identify these parameters, more calculations must be carried out at every time that the circuit is degraded. So, it is just $\Delta t_{r}$ (energy relaxation time difference), $\tau$ (time constant), and the amplitude of the measured signal that are used in this paper to set the fuzzy rules. However, as discussed earlier in this section, $\tau$ might not be an ideal parameter for $R L C$ circuits, but it does present a similar meaning as an attenuation factor $[\alpha=1 /(2 \tau)]$. Fig. 9 presents the proposed fuzzy-based RUL estimator (see Fig. 9).

\section{Resolving Uncertainties}

In reality, the monitored signals are often affected by noise and ripples, thus requiring further care to be provided, such as filtering in order to reduce uncertainties for accurate RUL estimation. In this regard, instead of making direct decisions based on monitored signals, the monitored signals can be shifted in the first-in first-out (FIFO) stack one by one, and the mean value of available data in the FIFO could thus be used for the RUL estimation (see Fig. 9). FIFO has a fixed storage length so that shifting a new sample to the FIFO will release the sample that had already been shifted into the stack at the earliest time. The mean value of FIFO captures the trend of signals and eliminates noise, unwanted information, and uncertainties. The estimation of RUL system reasoning will be enhanced as a result of FIFO. The length of FIFO has had a great impact on eliminating noise, but it normally should not take too long to lose trend of system degradation. Additionally, implementing a mean value on the stored data in the FIFO may add a dc/mean value to the 


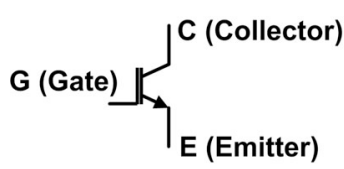

(a)

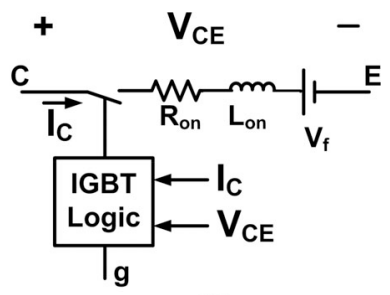

(b)
Fig. 10. IGBT model for simulation.

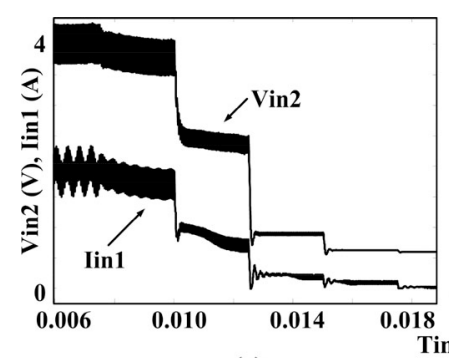

(a)

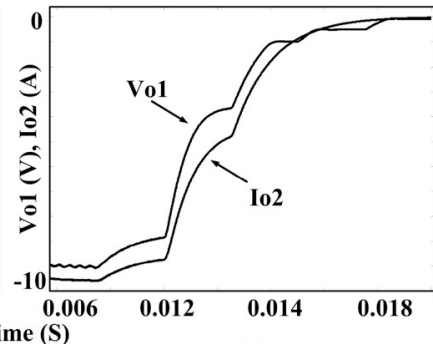

(b)
Fig. 11. Changes in input-output signals due to degradation in IGBTs.

estimated RUL. As the dc value is constant (i.e., it is meaningless information), it can be easily eliminated from the RUL.

\section{Simulation ReSUlts, Discussion, AND VALIDATION}

All simulations and experiments are conducted by OrCAD 16.6. Certain values used for Cuk converter devices as well as all the equations depicted in [36] refer to all the simulations in this paper. Only IGBT failure, which is the component that mostly experiences degradation during real time, is included in the simulation, and simultaneously, all other components are assumed to behave as nonaged devices (in all simulations) and that impedance of load remains constant. This degradation is simulated by changes experienced in the IGBT's forward resistor $R_{\text {on }}$, which is utilized in the MATLAB Simulink model of IGBT (see Fig. 10). As a switch, IGBT has two working modes-onstate and off-state-that connect and disconnect $R_{\text {on }}$ from the rest of the circuit, respectively. Hence, the IGBT's voltage across the collector-emitter is dropped down for the on-state mode as $R_{\text {on }}$ has values less than $1 \Omega$. On the other hand, the IGBT's current through the collector-emitter is dropped down for an off-state mode. Any variation in $R_{\text {on }}$ due to degradation causes the on-state and off-state values of the IGBT ( $V_{\text {ce }}$ and $\left.I_{\text {ce }}\right)$ to be changed. Hence, $V_{\text {ce-on-state }}$ and $I_{\text {ce-off-state }}$ could be known as one of the best parameters to monitor IGBT's failure.

\section{A. Parameters for System-Level Prognostics}

OrCAD 16.6 presents the simulation results for the Cuk converter, and its dual circuit shown in Fig. 11, which refer to the circuits in Fig. 1(c) and (d). In regard to these figures, it can be established that $V_{o 1}$ has the same trend as $I_{o 2}$; and the same is true for $I_{\mathrm{in} 1}$ and $V_{\mathrm{in} 2}$. These, in turn, reflect the similarities encountered within the transfer functions such as $Z, Y, A_{v}$, and

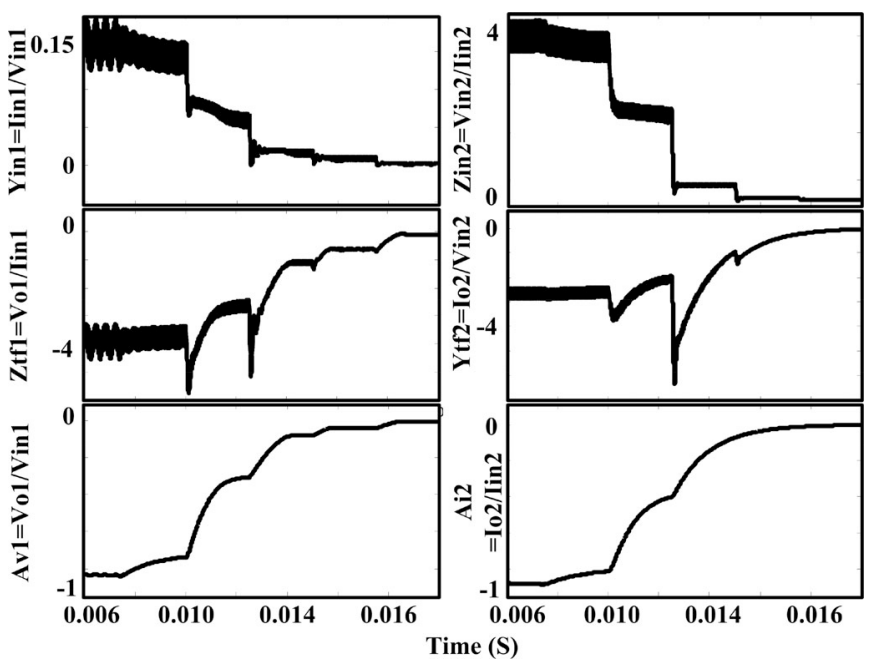

Fig. 12. Changes in transfer functions due to degradation.

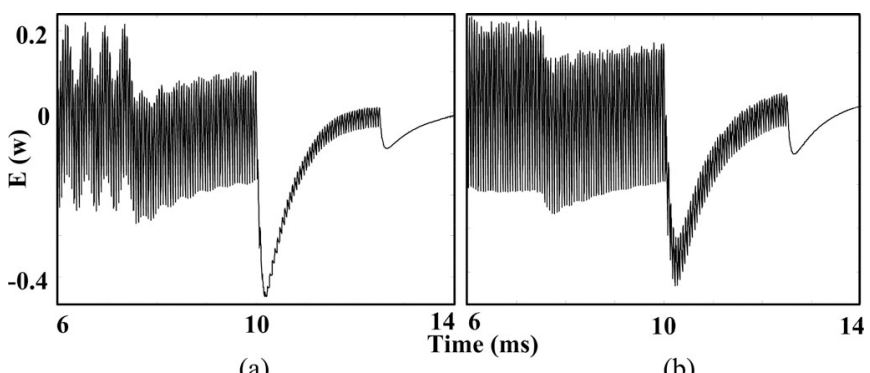

(a)

(b)

Fig. 13. (a) Energy in $C_{o}$ of Cuk converter in Fig. 1(c); b) energy in $L_{o}$ for dual circuit of Cuk in Fig. 3 .

$A_{i}$ shown in Fig. 12. Degradation was added to our simulations by changing the IGBT's parameters, such as $R_{\text {on }}$, in such a way that a trend of failure in Fig. 4 could be obtained from the IGBT model in Fig. 10. To run the simulations faster, we intend to have all 4500 cycles shown in Fig. 4 in just 25 ms. The result from this mapping is that the degradation will be accelerated in such a way that the first degradation will be experienced around $8 \mathrm{~ms}$ after exciting the circuit with a step function $u(t)$; however, the threshold needed to estimate whether the IGBT has aged enough to incur damage in an earlier time is around $10 \mathrm{~ms}$. The same lifetime and threshold can be expected from energy transfer and power of $C_{o}$ and $L_{o}$, respectively, shown in Fig. 13 for Cuk and its dual circuit. As illustrated in Fig. 13, due to degradation, energy will not be sufficiently transferred to the circuit. This leads us to acknowledge that the health state of a circuit can be just as well understood from knowing the state equations of a circuit, whereby energy signals from storing elements (capacitors and inductors) are used as state variables.

The timings shown from these simulations are not realistic as we accelerated the aging mechanisms in the simulation. Nevertheless, it does clearly prove that the concept of the circuits with duality relationships or even with similarities in components and topologies may also have similar prognostic models. Every signal, energy, and transfer function monitored in the figures 
has similar information with regard to the effects of degradation of a circuit. However, as a result of the switching scheme of IGBT and energy transfer in capacitors and inductors rapidly changing, some of these parameters are affected by small ripples. However, in most cases, the same trend can be found in all these signals.

As shown in Fig. 12, $A_{v 1}$ and $A_{i 2}$ seem to be the best for utilizing various parameters such as $\tau$ and $\alpha$, which are required to calculate RUL. The other transfer functions and signals are viewed as noisy data, thus requiring further care to be provided such as filtering in order to reduce uncertainties; hence, FIFO presented in Fig. 9 would be needed to reduce uncertainties caused by them. $A_{v 1}$ and $A_{i 2}$ in Fig. 12 show that attenuation and damping factors are increased while the IGBT is aged. The result of the IGBT degrading means that the circuit will settle down faster to its usual steady-state mode but have a different steady-state value. This further means that the duration related to the transition mode will be shortened. While the degradation is progressing, it does seem that the degradation appears to be in a trend of several different states rather than the signals slightly increasing or decreasing over time. In other words, there are several degradation domains related to the measured signals during which the signals suddenly drop or rise. The reason for this sudden change can be found in the structure of IGBT and its failure mechanism. So IGBT is constructed on a die, and then a connection is made to the package using a number of wire bonds. As a result of degradation, some of these wires are lifted off, but they are not lifted off at the same time. As soon as some of these wires are lifted off, a new degradation domain is established from the trends found in the signals. Because of this effect, the internal resistor of the IGBT in the model $\left(R_{\text {on }}\right.$ shown in Fig. 10) is changed, which leads to $\alpha$ changing as well. We use the same equation of $\tau$, presented in (5) and (6), to charge and discharge the circuit. This is done to measure $\alpha$ from $V_{o 1}$ and $I_{o 2}$ in Fig. 12 while knowing that $\alpha=1 /(2 \tau)$ is for $R C$ or $R L$ circuits. These are equations used for calculating voltage across any capacitor while it is charging or discharging [22]. Hence, this might not be completely correct as the circuit from the perspective of this signal cannot be simplified as parallel or as a series of $R C$, but it appears to be sufficient to fulfill the proposed RUL estimation algorithm where fuzzy knowledge base is employed

$$
\begin{aligned}
& V_{o 1}(t)=V_{\mathrm{st}}\left(1-e^{-t / \tau}\right)+V_{o 1}(0) e^{-t / \tau} \\
& V_{o 1}(t)=V_{\mathrm{st}}+\left(V_{o 1}(0)-V_{\mathrm{st}}\right) e^{-t / \tau} .
\end{aligned}
$$

where $V_{\mathrm{st}}$ is the steady-state value of $V_{o 1}$ for a particular degradation domain that is progressed and $V_{o 1}(0)$ is the initial value for the $V_{o 1}$ at the beginning of this particular degradation domain. Table II provides values of $\tau$ and $\alpha$ for each degradation domain. These values are calculated from the initial and steadystate values, which are directly measured from $V_{o 1}$ in Fig. 12, and then $\tau$ is calculated using (5) and (6) and $\alpha=1 /(2 \tau)$. The values show that the time constant is generally decreased while attenuation is increased.
TABLE II

Time Constant and ATtENUATION FaCtor For EACH DEgRadation DOMAIN

\begin{tabular}{lcccccc}
\hline \hline & Domain 1 & Domain 2 & Domain 3 & Domain 4 & Domain 5 & Domain 6 \\
\hline $\boldsymbol{\tau}$ & 1080 & 630 & 360 & 270 & 90 & 140 \\
$\boldsymbol{\alpha}$ & 0.000463 & 0.00074 & 0.00139 & 0.0018 & 0.0055 & 0.0036 \\
\hline \hline
\end{tabular}

\section{B. RUL Estimation}

The process can be simplified by using $A_{v 1}$ and $A_{i 2}$ for the RUL estimation by applying fuzzy logic techniques to estimate the remaining lifetime of a circuit, as shown from the algorithm in Fig. 7. All the input and output membership functions are set in Gaussian form with input fuzzy values as s small, medium, normal, transient, big $\}$ and output fuzzy values as $\{$ health_state_1, health_state_2, health_state_3, health_state_4, health_state_5\}. There are three sets of fuzzy inputs, the attenuation factor, the amplitude of $V_{o 1}$, and lastly energy relaxation time difference $\left(\Delta t_{r}\right)$. The output of fuzzy logic is based on the RUL estimation in a fuzzy set. This means that input fuzzy values refer to changes in the trend of $A_{v 1}$ and $A_{i 2}$. Output fuzzy values refer to the life state of the circuit such as young for health_state_1 and aged for health_state_5. We also use the number of adverbs and adjectives to address $10 \%$ and $90 \%$ confidence levels in life estimation based on the time needed for $85 \%$ and $95 \%$ energy loss (see Fig. 8). Rules in the fuzzy knowledge base are in the following form:

If $V_{o 1}(C)$ is $X(i)$ and $\alpha(C)$ is $Y(j)$ and $\Delta t_{r}(C)$ is $Z(k)$, then $\operatorname{RUL}(C)$ is Life_State $(m)$

where $C$ is the index of a measurement point; $i, j, k$, and $m$ are the indices of selected membership functions from 1 to 5 for the input and output fuzzy variables of $V_{o 1}, \alpha, \Delta t_{r}$, and RUL. We also had the following fuzzy rule in cases where $V_{o 1}$ remains almost the same in steady-state mode and $\alpha$ has a small fuzzy value. This rule means that the life state remains unchanged.

If $V_{o 1}(C)$ is normal and $\alpha(C)$ is small and $\Delta t_{r}$ is more or less small, then RUL $(C)$ is Life_State(-).

Here, Life_State(-) means that whatever life state RUL had at a previous cycle time will be valid for the current cycle time; however, it may be fired with different fuzzy values of the same life state which depends on the fuzzy values. RUL is estimated using the MAX fuzzy function which is applied on triggered fuzzy values. The MAX fuzzy function selects the maximum fuzzy value among the fired membership functions. This allows the RUL to be estimated in a fuzzy form, and therefore needs to be defuzzified. During the defuzzification stage, RUL is also scaled up so that the integration of the estimated RUL (in fuzzy form) reflects the maximum life cycle of the circuit.

As explained in Section VI, parameter $\alpha$ requires understanding the duration of each degradation domain; however (when samples are fed to the simulation one by one), the samples that cover the whole duration of the degradation domain are not available during the process of RUL estimation. A solution to this problem is to employ $\Delta V_{o 1} / \Delta t$ instead of $\alpha$ that represents slop of $V_{o 1}$ that is time constant $\tau$. To avoid spike affects while 


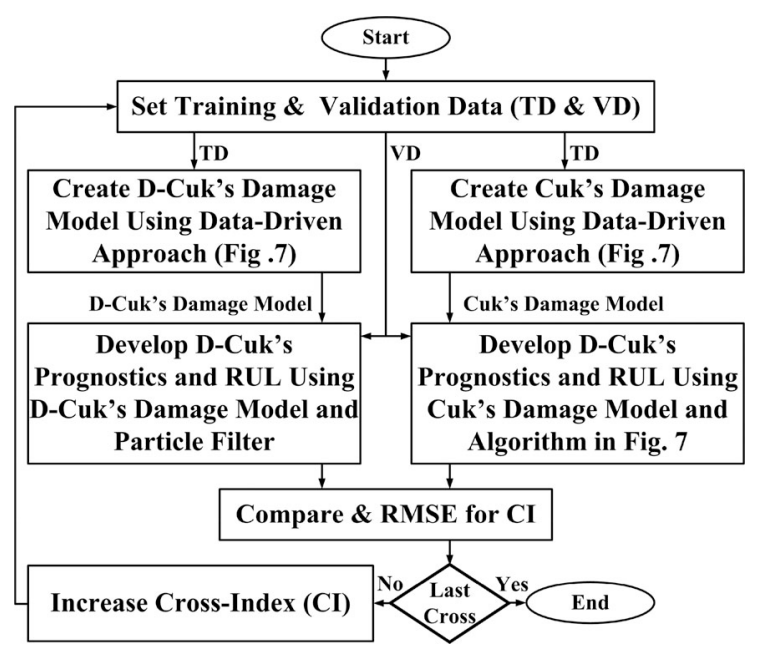

Fig. 14. Validation approach.

improving reasoning, a FIFO with storage length of 10 samples is used (see Fig. 9). $V_{o 1}(t)$ is obtained from the mean value of first half of FIFO, and $V_{o 2}(t-1)$ is obtained from the second half of FIFO. $\alpha$ is fuzzified along with amplitude of $V_{o 1}(t)$; and then, these are fed to the fuzzy knowledge base. Thus, $\alpha$ will be obtained from (7), assuming that $\alpha=1 /(2 \tau)$

$$
\alpha(t)=\frac{1}{2 \tau}=\left(\frac{\sum_{1}^{5} V_{o 1}-\sum_{6}^{10} V_{o 2}}{10}\right) .
$$

\section{VALIDATION}

Validating a prognostic model requires sufficient statistical data from a circuit that is aged under various operating conditions. This is needed to create a statistical model for a circuit's failure mechanisms. To enhance this model with such statistical behavior, a binominal distribution might be constructed using a collection of $n$ trials from field data. So, for the purpose of this paper, we were limited to four aging data sets of IGBTs from Nottingham University. The cross-validation technique is employed here to validate our concepts, which relates to the prognostics of the Cuk converter $\left(P_{\mathrm{Cuk}}\right)$ being applied to its dual circuit $\left(P_{D-\mathrm{Cuk}}\right)$. Fig. 14 shows an algorithm for the cross validation technique employed in this paper. The proposed technique is employed to develop a damage model for Cuk converter based on data-driven approaches (see Fig. 6) and from using three sets of IGBT failure data obtained from Nottingham University to present failure in the Cuk converter. The damage model is then used to develop a prognostic model for the DCuk converter using the proposed techniques while the fourth data set is used as validation data. Based on the measurement points given in the validation data, collections of Dirac functions excite the circuit; and for each Dirac, energy relaxation time $\left(t_{r}\right)$ is measured. $\Delta t_{r}$ is calculated based on nominal $t_{r}$ from the Cuk converter and actual $t_{r}$ from D-Cuk. $\Delta t_{r}$, attenuation, and the current of the load (from D-Cuk) are used by the fuzzy RUL estimator to estimate the RUL of the Cuk converter's dual circuit. This process is repeated four times based

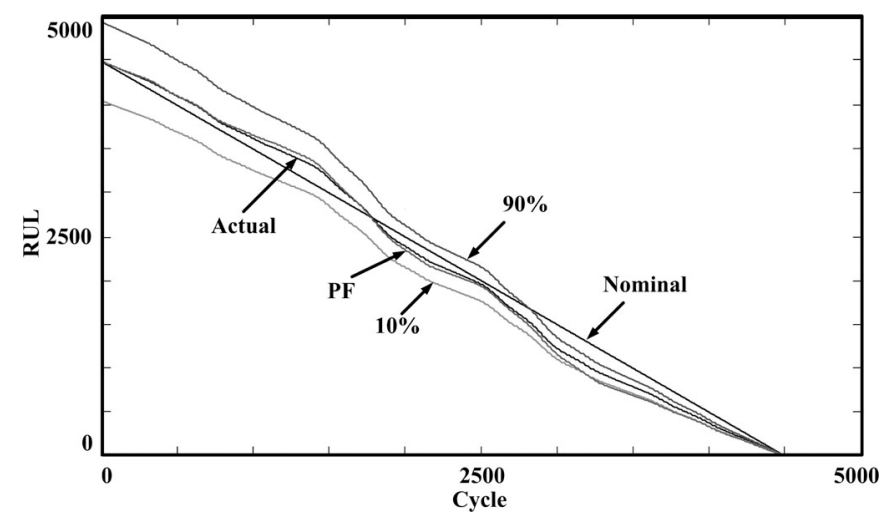

Fig. 15. Resulting RUL after testing prognostic model with data test.

on the cross-validation technique, so that each time, the Cuk converter's damage model is recreated using three new sets of IGBT failure data (train data); and then, the RUL of D-Cuk is estimated using the remaining data set as validation data.

For the purpose of validation and comparison with other techniques, a model-based prognostic is also created for the dual circuit of the Cuk converter using a particle filter. The results from each iteration of the cross validation loop, which is based on the data-driven approach (see Fig. 6), is compared with the prognostics results of the second approach. To pursue such a validation process, a failure model is considered for the dual circuit of the Cuk converter [see(8)], which is based on simulation results given in Fig. 11

$$
I_{o 2}=\sum_{i=1}^{6}\left(P_{i 1}\left(1-e^{\left(t-P_{i 2}\right) / P_{i 3}}\right)+P_{i 4}\right)
$$

where $I_{o 2}$ is the current of the load, $t$ is the time index (when the sample has been taken), $p_{i 1}$ to $p_{i 4}$ are parameters used by the particle filter estimation algorithm, and $i=\{1,2, \ldots, 6\}$ is the number of clusters detected in the IGBT's failure data using the $K$-means and silhouette clustering tools of MATLAB. These parameters are related attenuation and energy relaxation time of circuit, and are updated by its likelihood during the prognostic process of IGBT accordingly [19]. Equation (8) has been used to develop a damage model for the dual circuit of the Cuk converter and also for the estimation algorithm based on the particle filter. We employed the same technique and programming codes as presented in [19] to develop system-level model-based prognostics.

The estimated RUL using the algorithm given in Fig. 6 is compared with the estimated RUL using the particle filter for D-Cuk in Fig. 15. In this figure, the RUL's confidence levels accounted based on $85 \%$ and $95 \%$ energy loss are also represented. The actual RUL for D-Cuk is estimated based on $90 \%$ energy loss. Ideally, it is expected that the life of a circuit is decreased as a negative ramp as nominal RUL shown in Fig. 15. However, our simulation results show that the RUL is slightly wavy. The root-mean-square error (RSME) and the mean absolute error (MAE) performance function of $1.9555 \mathrm{e}+005$ and 48.9259 , respectively, accounted for the error between the 
actual RUL of D-Cuk estimated using the proposed algorithm and the particle filter $(\mathrm{PF})$.

We realized that if a degradation profile is used for the Cuk converter; such that it is converted to a malfunction profile for its dual circuit so that the IGBTs in both circuits always remain in dual forms. Then, a duality relationship would be established between the transferred functions of these two circuits. For instance, $Z_{c}(t)$ is equal to $Y_{\mathrm{dc}}(t)$, where $Z_{c}(t)$ is impedance of Cuk and $Y_{\mathrm{dc}}(t)$ is admittance of D-Cuk. This is because as the degradation profile changes the IGBT of Cuk toward an open circuit; its malfunction profile also changes the IGBT of dual circuit toward a short circuit.

If the malfunction profile for the dual circuit of Cuk is not extracted from the degradation profile of the Cuk circuit, then $Z_{c}(t)$ will not be identical to $Y_{\mathrm{dc}}(t)$. We can, however, conclude that if the whole process is repeated for a number of different degradation and malfunction profiles and that the mean value of $Z_{c}(t)$ and $Y_{\mathrm{dc}}(t)$ are used for comparison; leads to meaningful similarity patterns to be found between $Z_{c}(t)$ and $Y_{\mathrm{dc}}(t)$. $Z_{\mathrm{mc}}(t)$ can be used for the mean value of $Z_{c}(d, t)$ and $Y_{\mathrm{mdc}}(t)$ can be used for the mean value of $Y_{\mathrm{dc}}(d, t)$, in situations where $\mathrm{m}$ refers to the mean value. Both $Z_{\mathrm{mc}}(t)$ and $Y_{\mathrm{mdc}}(t)$ can be used as prognostic models for the Cuk converter and its dual circuit. However, these two transfer functions are not exactly identical, but they would be more similar to one another if the process that is required to be executed to obtain the functions is repeated for various numbers of degradation and malfunction profiles for both circuits. The accuracy of the prognostic model can be further increased by implementing more intelligent algorithms that use stochastic, neural network, fuzzy and other techniques instead of a simple mean value functions. By implementing such intelligent algorithms also reflects the future aim and direction of our research. We should not forget that prognostics have always been a way to estimate the life cycle of devices and systems within different confidence levels. Confidence levels provide assurance that we can comfortably rely on the performance of an aged system. The critical point to consider is that the accuracy of prognostic models has always been under doubt and remains to be within margins of confidence levels. In summary, by using the prognostic model of a system for other systems where similarities in their properties (like duality) are found, would provide us with a more accurate and reliable representation of the state and condition of a system. This is assuming that the prognostics are developed from an adequate number of degradation profiles, and consist of the right minimum and maximum confidence levels.

The concept presented in this paper is applicable for systems with duality connection when both a circuit and its dual have the same components connected with different topologies. The reason for such a limitation is to guarantee that both circuits will experience the same degradation mechanisms under different working conditions. As a case study, for instance, both Cuk and D-Cuk converters are comprised from resistor, capacitors, inductances, IGBT, and diodes, while having dual topologies. Further research is needed to be conducted for situations where dual circuits are not comprised from the same components. We are going to carry on with our research to create a unified prognostic model that predicts the future damage and life state of all 33 different topologies of dc-to-dc converters with just one model.

\section{CONCLUSION}

In conclusion, this paper illustrates that the prognostics of systems that share similar properties in the form of duality can be applied to one another. This prognostic model is developed in the form of a time-dependant transfer function based on the degradation mechanisms related to a system's components, the values are subsequently altered over a certain period of time. A duality connection found within the degradation and malfunction profiles of a system can be established by having the prognostics assigned to a system's property. So, if we were to consider that the components of a system are aged, this will mean that their dual components in the dual circuit will be faced with malfunction. The accuracy of the developed prognostic model highly depends on the number of degradation profiles available; and the methodology used to train the time-dependant transfer function. The accuracy of this model can be guaranteed and expressed via the minimum and maximum confidence levels. However, this approach only applies to the Cuk converter and its dual circuit. However, it appears that the same technique can be applied for systems that have slightly similar mechanisms, components, properties, topologies, and degradation. Therefore, further research needs to be conducted for systems that are not in dual forms, especially for the purposes of exploring how the prognostic model of a system could be mapped to the prognostic model of another system.

The positive aspects of pursuing such a technique have been emphasized in the implementation stage of the inference engine for SLR and the SIPR. It additionally provides us with the required facility to transfer degradation knowledge and experiences between systems. This means that it would be much faster to develop prognostics for complex systems, like HDSs, which are used for certain applications such as aircraft while also decreasing the costs related to the accelerated aging tests and preparing degradation profiles. We essentially intend on pushing forward with our research, in order to apply this technique to developing a prognostic inference engine for various topologies of dc-to-dc converters.

\section{ACKNOWLEDGMENT}

The authors would like to sincerely thank Prof. C. M. Johnson and Dr. P. Evans from the Power Electronics, Machines and Control Group, University of Nottingham, for the contribution of failure data of the IGBTs and the power cycling test rig configuration.

\section{REFERENCES}

[1] I. K. Jennions, Integrated Vehicle Health Management Perspectives on an Emerging Field. Pittsburgh, PA, USA: SAE International, 2011, pp. $100-110$.

[2] A. El-Sayed and M. ElHelw, "Distributed component-based framework for unmanned," in Proc. IEEE Int. Conf. Inform. Autom., Shenyang, China, Jun. 2012, pp. 45-50. 
[3] W. Wenbin and M. Carr, "A stochastic filtering based data driven approach for residual life prediction and condition based maintenance decision making support" in Proc. IEEE Prognostics Syst. Health Manage. Conf., 2010, pp. 1-10.

[4] I. K. Jennions, Integrated Vehicle Health Management the Technology. Pittsburgh, PA, USA: SAE International, 2013, pp. 139-154.

[5] B. T. Thumati, M. A. Feinstein, and S. Jagannathan, "A model-based fault detection and prognostics scheme for Takagi-Sugeno fuzzy systems," IEEE Trans. Fuzzy Syst., vol. 22, no. 4, pp. 736-748, Aug. 2014.

[6] L. Xiaoxiong, S. L. Yuan, H. Zhiyi, and C. Kang, "Research of actuator fault health management method based on neural network," in Proc. IEEE Int. Conf. Soft Comput. Pattern Recog., Dalian, China, Oct. 14-16, 2011, pp. 227-230.

[7] B. W. Williams, "Generation and analysis of canonical switching cell DCto-DC converters," IEEE Trans. Ind. Electron., vol. 61, no. 1, pp. 329-346, Jan. 2014

[8] J. Li, "Optimal fault diagnosis based on duality principle for linear systems," in Proc. Control Decision IEEE Conf., Jul. 2008, pp. 573-577.

[9] M. Daigle and K. Goebel, "Model-based prognostics under limited sensing," in Proc. IEEE Aerosp. Conf., Mar. 2012, pp. 1-12.

[10] C. Chen and M. Pecht, "Prognostics of lithium-ion batteries using model based and data-driven methods," in Proc. IEEE Prognostics Syst. Health Manage. Conf., Beijing, China, 2012, p. 6.

[11] T. Sreenuch, A. Alghassi, S. Perinpanayagam, and Y. Xie, "Probabilistic Monte-Carlo method for modelling and prediction of electronics component life," Int. J. Adv. Comput. Sci. Appl., vol. 5, no. 1, pp. 96-104, 2014.

[12] S. Sarkar, X. Jin, and A. Ray, "Data-driven fault detection in aircraft engines with noisy sensor measurements," J. Eng. Gas Turbines Power, ASME, vol. 133, pp. 1-10, Aug. 2011.

[13] L. Jianhui, M. Madhavi, K. Pattipati, Q. Liu, M. Kawamooto, and S. Chigusa, "Model-based prognostic techniques applied to suspension system," IEEE Trans. Syst, Man Cybern., Part A: Syst. Humans, vol. 38, no. 5, pp. 1156-1168, Sep. 2008

[14] P. Shetty, D. Mylaraswamy, and T. Ekambaram, "A hybrid prognostic model formulation system identification and health estimation of auxiliary power units," in Proc. IEEE Aerosp. Conf, Big Sky, MT, USA, Mar. 2006, pp. $1-10$.

[15] A. K. Garga, K. T. McClintic, R. L. Campbell, Y. Chih-Chung, M. S. Lebold, T. A. Hay et al., "Hybrid reasoning for prognostic learning in CBM systems," in Proc. IEEE Aerosp. Conf., Big Sky, MT, USA, Mar. 2001, vol. 6, pp. 2957-2969.

[16] A. Alghassi, S. Perinpanayagam, and I. Jennions, "A simple state-based prognostic model for predicting remaining useful," presented at the 15 th Eur. Conf. Power Electron. Appl., Lille, France, 2013.

[17] J. M. Thebaud, E. Woirgard, C. Zardini, and C. K. H. Sommer, "High power IGBT modules: Thermal fatigue resistance evaluation of the solder joints," in Proc. IEEE Int. Workshop Integr. Power Packag., IWIPP, 2000, pp. 79-83.

[18] V. A. Sankaran, C. Chen, C. S. Avant, and X. Xu, "Power cycling reliability of IGBT power modules," in Proc. IEEE Ind. Appl. Conf., 32nd IAS Annu. Meeting, IAS, pp. 1222-1227, Oct.5-9, 1997.

[19] D. An, J. Choi, and N. Kim, "Prognostics 101: A tutorial for particle filter-based prognostics algorithm using MATLAB," in Elsevier, Reliability Engineering and System Safety, vol. 115, pp. 161-169, 2013.

[20] M. E. Orchard and G. J. Vachtsevanos; "A particle-filtering approach for on-line fault diagnosis and failure prognosis," Trans. Institute Meas. Control, vol. 31, no. 3-4, pp. 221-46, 2009.

[21] P. Li, R. Goodall, and V. Kadirkamanathan, "Parameter estimation of railway vehicle dynamic model using Rao-Blackwellised particle filter," presented at the Eur. Control Conf., Cambridge, U.K., Sep 2003.

[22] L. O. Chua, C. A. Desoer, and E. S. Kuh, Linear and Nonlinear Circuits. New York, NY, USA: McGraw-Hill, 1st ed., Mar. 1987.

[23] D. Y. Gao, Duality Principles in Nonconvex Systems: Theory, Methods and Applications. Norwell, MA, USA: Kluwer, 2000.

[24] G. E. Sharpe and G. P. H. Styan, "Circuit duality and the general network inverse," IEEE Trans. Circuit Theory, vol. CT-12, no. 1, pp. 22-27, Mar. 1965.

[25] R. W. Erickson, "Synthesis of switched-mode converters," in Proc. IEEE Power Electron. Spec. Conf., 1983, pp. 9-22.

[26] D. Maksimovic, "Synthesis of PWM and quasi-resonant DC-to-DC power converters," Ph.D. dissertation, California Inst. Technol., Pasadena, CA, USA, 1989.
[27] Y. S. Lee, "A systematic and unified approach to modeling switches in switch-mode power supplies," IEEE Trans. Ind. Electron., vol. IT-32, no. 4, pp. 445-450, Nov. 1985.

[28] A. K. S. Bhat and F. D. Tan, "A unified approach to characterization of PWM and quasi-PWM switching converters: Topological constraints, classification, and synthesis," IEEE Trans. Power Electron., vol. 6, no. 4 pp. 719-726, Oct. 1991

[29] M. Ogata and T. Nishi, "Topological criteria for switched mode dcdc converters," in Proc. Int. Symp. Circuits Syst., May 2003, vol. 3, pp. $184-187$.

[30] D. H. Wolaver, "Basic constraints from graph theory for DC-to-DC conversion networks," IEEE Trans. Circuit Theory, vol. CT-19, no. 6, pp. 640-650, Nov. 1972

[31] Y. Berkovich, A. Shenkman, A. Loinovici, and B. Axelrod, "Algebraic representation of DC-DC converters and symbolic method of their analysis," in Proc. IEEE 24th Convent. Elect. Electron. Eng., Eilat, Israel, 2006 pp. 47-51.

[32] M. Ogata and T. Nishi, "Topological conditions for passive switches in switching converters," in Proc. 18th IEEE ECCTD, 2007, pp. 898-901.

[33] T. Nishi, T. Oghishima, and M. Ogata, "Topological conditions on switched mode DC-DC converters," in Proc. ITC-CSCC, Jul. 2002, pp. 1129-1132.

[34] R. Severns, "Switch mode converter topologies make them work for you," Intersil, Inc., Milpitas, CA, USA, Appl. Bull. A035, 1980.

[35] B. W. Williams, "Generation and analysis of canonical switching cell DCto-DC converters," IEEE Trans. Ind. Electron., vol. 61, no. 1, pp. 329-346, Jan. 2014.

[36] F. J. Rytkonen and R. Tymerski. Modern control regulator de sign for $d c-d c$ converters [Online]. Available: http://web.cecs.pdx.edu/ $\sim$ tymerski/ece451/Cuk_Control.pdf

[37] C. S. Kulkarni, G. Biswas, and X. Koutsoukos, "Integrated diagnostic/prognostic experimental setup for capacitor degradation and health monitoring," presented at the IEEE Conf., Processing Autotestcon, Orlando, FL, USA, Sep. 2010.

[38] C. S. Kulkarni, J. R. Celaya, G. Biswas, and K. Goebel, "Prognostics of power electronics, methods and validation experiments," presented at the IEEE Conf. Autotestcon, Anaheim, CA, USA, 2010.

[39] M. Alioto, G. Palumbo, and M. Poli, "Analysis and modeling of energy consumption in RLC tree circuits", IEEE Trans. Very Large Scale Integr. Syst., vol. 17, no. 2, pp. 278-291, Feb. 2009.

[40] P. Miettinen, M. Honkala, J. Roos, and M. Valtonen, "PartMOR Partitioning-based realizable model-order reduction method for RLC circuits," IEEE Trans. Comput.-Aided Design Integr. Circuits Syst., vol. 30, no. 3, pp. 374-387, Mar. 2011.

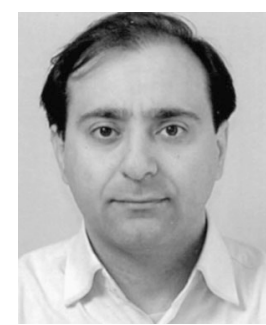

Mohammad Samie received the Ph.D. degree in advanced electronics from the University of the West of England (UWE), Bristol, U.K., in 2012.

He was involved in research on self-healing cellular architectures for biologically inspired highly reliable electronic systems funded by EPSRC, a joint research establishment between UWE and the University of York. He is currently a Research Fellow for Integrated Vehicle Health Management (IVHM) Centre, which is jointly funded by Boeing, Cranfield University, and a number of other industrial partner to conduct research on system health management, power electronics, and selfhealing systems.

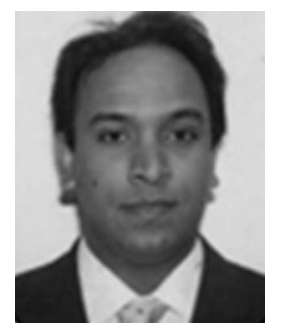

Suresh Perinpanayagam (M'12) received the M.Eng. and D.Phil. degrees from Imperial College London, London, U.K., in 2004.

$\mathrm{He}$ is a Lecturer at the Integrated Vehicle Health Management (IVHM) Centre, Cranfield University, Bedford, U.K., where he heads the Electronics Prognostics Group, which works on highly accelerated life testing, electrothermal modeling, thermomechanical modeling, reliability and failure assessment capabilities for renewable energy systems, automotive, aerospace, and rail applications. This laboratory develops data-mining techniques for anomaly detection, diagnostics, prognostics, and predictive maintenance for power electronics from the aforementioned industry sectors. 


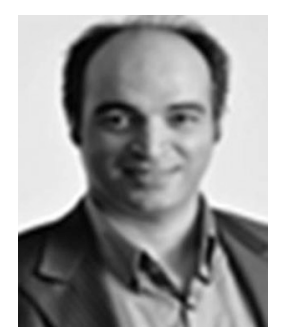

Alireza Alghassi (S'14) received the B.Sc. degree in electrical power engineering from Iran and Control System Engineering, Sheffield University, Sheffield, U.K., and the M.Sc. degree in power electronic engineering from Nottingham University, Nottingham, U.K. He is currently working toward the Ph.D. degree in the School of Applied Sciences, IVHM Centre, Cranfield University, Bedford, U.K.

$\mathrm{He}$ is a Researcher at IVHM Centre, Cranfield University.

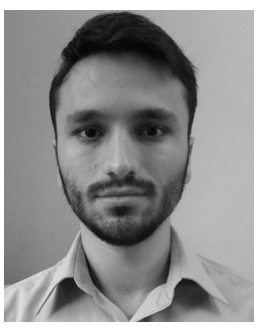

Amir M. S. Motlagh is currently working toward the Ph.D. degree at Westminster University, London, U.K.

His current research interests include applying semantic-based computational methods to effectively and efficiently search and retrieve systems' information. He has a deep understanding of how to utilize semantic technologies, clustering, and classification techniques for conducting similarity measurement and indexing within various fields.

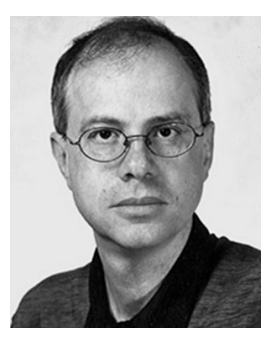

Epaminondas Kapetanios received the B.Sc. degree (Hons.) in statistics and informatics from Athens University of Economics and Business, Greece, in 1987 , the master's degree in information systems from the Karlsruhe Institute of Technology, Karlsruhe, Germany, in 1990, and the Ph.D. degree in computer science from the Swiss Federal Institute of Technology (ETH), Zurich, Switzerland, in 2001.

$\mathrm{He}$ is currently a Senior Lecturer at the University of Westminster, London, U.K. He has spent more than 15 years in research related to Information Science and Systems, with particular emphasis on both algorithmic thinking (also known as "the spirit of computing"), design and implementation, and on data and knowledge engineering aspects, in order to provide a more systemic approach to problem solution strategies for scientific and technological challenges in ICT of the 21 st century. The research contributions span over a variety of topics such as information retrieval, text mining, natural language processing, query languages, Web search and intelligence, pattern search and recognition. More specific, all algorithmic and data/knowledge engineering approaches have been informed by the intriguing question of how a machine can understand and process semantics and context, a key step in machine intelligence. He has authored or coauthored in peer-reviewed journals and conferences. He has also authored the book Natural Language Processing: Semantic Aspects (CRC Press).

Dr. Kapetanios was the General Chair and the Programme Committee Chair, respectively, for conferences such as NLDB 2008 and ACM MEDES 2009. 
2019-12-02

\section{Developing prognostic models using duality principles for DC-to-DC converters}

Samie, Mohammad

IEEE

Samie M, Perinpanayagam S, Alghassi A, et al., (2015) Developing prognostic models using duality principles for DC-to-DC converters. IEEE Transactions on Power Electronics, Volume 30, Issue 5, May 2015, pp. 2872-2884

https://doi.org/10.1109/TPEL.2014.2376413

Downloaded from Cranfield Library Services E-Repository 